\title{
Quelques caractéristiques organoleptiques et valeurs nutritionnelles de la viande de Thryonomys swinderianus (Temminck, 1827) élevés en captivité et nourris avec deux types d'aliments
}

\author{
Soronikpoho $\mathrm{SORO}^{1 *}$, Yolande AKE ASSI${ }^{2}$, Beh TRAORE ${ }^{3}$ et Agathe FANTODJI \\ ${ }^{1}$ Université Peleforo Gon Coulibaly, Institut de Gestion Agropastorale, BP 1328 Korhogo, Côte d'Ivoire. \\ ${ }^{2}$ Laboratoire Central pour l'Hygiène Alimentaire et l'Agro-Industrie (LCHAI), 04 BP 612 Abidjan 04, \\ Côte d'Ivoire. \\ ${ }^{3}$ Université Nangui Abrogoua, Département des Sciences de la Nature, BP 801 Abidjan 02, Côte d'Ivoire. \\ *Auteur correspondant; E-mail : soronikpohosoro@gmail.com, Tel : (+225) 07514612
}

\section{RESUME}

L'aulacodiculture est une option privilégiée à la fourniture de protéines animales. Cette étude a été menée dans le but de déterminer l'effet d'un aliment granulé sur les caractéristiques organoleptiques et biochimiques de leur viande. Ainsi, un lot d'aulacodes a été nourri au granulé tandis que l'autre nourri au fourrage avec complément alimentaire (aliment classique). Après un abattage normal, les membres antérieurs et postérieurs des animaux ont été prélevés. La viande des membres postérieurs a été retenue pour l'analyse organoleptique (couleur, texture, jutosité, flaveur, tendreté) par 12 panelistes en trois répétitions. La viande des membres antérieurs a servi à la détermination de la matière sèche, des protéines brutes, de la matière grasse et des cendres brutes. Ces travaux montrent que la viande d'aulacode est juteuse, tendre avec une texture grossière et une flaveur prononcée quel que soit le type d'aliment. Le profil sensoriel montre une différence tant au niveau de la couleur qu'au niveau de la jutosité et de la texture. Quant aux paramètres biochimiques, aucune différence significative n'a été observée $(\mathrm{p} \geq 0,05)$. Il convient de retenir que la viande d'aulacodes nourris au granulé a été plus appréciée tant pour la couleur, la texture, la jutosité que pour la flaveur.

(C) 2020 International Formulae Group. All rights reserved.

Mots clés : Aulacodes, viandes, granulé, fourrage, Côte d'Ivoire.

\section{Some organoleptic characteristics and nutritional values and nutritionals values of meat of Thryonomys swinderianus, (Temminck, 1827) raised in captivity and fed with two types of food}

\begin{abstract}
Grasscutter farming seems to be a preferred option for the supply of proteins formerly derived from hunting. This study aimed at determining the effect of a granulated food on the organoleptic and biochemical qualities of grasscutter meat. Thus, three grasscutters were randomly selected from a batch of animals fed with a granulated food and the other three from the batch fed with the conventional food. After normal slaughter, the fore and hind limbs were removed. The meat of the posterior limbs was selected for organoleptic analysis by a panel of 12 consumers in three repetitions. The meat from upper limbs was used to evaluate the biochemical
\end{abstract}


parameters such as dry matter, crude protein, fat and raw ash. Results showed that the meat of the grasscutter was juicy, tender with a coarse texture and a pronounced flavor whatever the type of food. The sensory profile showed a significant difference in color, juiciness, and texture of meat. With respect to biochemical parameters, no significant difference was observed $(\mathrm{p} \geq 0.05)$. The type of food had no effect on biochemical parameters of grasscutter meat. However, the organoleptic parameters influenced significantly the level of appreciation of grasscutter meat.

(C) 2020 International Formulae Group. All rights reserved.

Keywords: Grasscutter, meat, granulated food, forage, Côte d'Ivoire.

\section{INTRODUCTION}

L'aulacode est un gibier très apprécié pour ces qualités organoleptiques et sa viande ne souffre d'aucun tabou (Niaga, 2002). Pour lutter contre l'exploitation abusive et assurer la sécurité alimentaire, tout en préservant la biodiversité et la santé des populations, sa domestication a été entreprise. Les travaux menés par différents chercheurs depuis des décennies ont abouti à l'élevage en captivité (Mensah et Ekoué, 2003). Ceci a engendré de nouveaux problèmes dont les difficultés d'affourragement en saison sèche. Cette période de soudure rend la tâche difficile aux éleveurs et influence négativement la reproduction des aulacodes. Pour résoudre ce problème, Traoré et al. (2009) et Soro et al. (2014) ont mis au point un aliment granulé à base de fourrage sec (foin) et de complément alimentaire. Les expérimentations menées sur les performances zootechniques montrent que les aulacodes nourris avec cet aliment ont des poids moyens et un rendement en carcasse significativement supérieurs à ceux des aulacodes nourris au fourrage. Cependant, l'impact de cet aliment granulé complet sur les seules performances zootechniques ne suffit pas pour apprécier sa qualité. Son impact sur les paramètres nutritionnelles et sensorielles sont aussi des critères importants intervenant dans la perception de qualité de la viande par les consommateurs. Certains auteurs dont Cassignol (2018) ont relevé d'autres facteurs propres à l'animal mais aussi du mode d'élevage et de l'alimentation sur la qualité de la viande.

L'objectif de ce travail était d'identifier les caractéristiques nutritionnelles et organoleptiques que préfèrent le consommateur pour les prendre en compte dans les schémas de sélection.

\section{MATERIEL ET METHODES}

Animaux expérimentaux

Six (06) aulacodes mâles âgés de sept à huit mois et pesant en moyenne $3,8 \pm 0,38 \mathrm{~kg}$ ont été utilisés. Ils ont été choisis en fonction du type d'aliment; soit trois pour l'aliment granulé et trois autres pour l'aliment classique. Après un abattage normal, il s'en est suivi le prélèvement des membres. Les membres postérieurs ont été utilisés pour l'analyse sensorielle et les membres antérieurs pour l'analyse biochimique. Afin de conserver leur intégrité, chaque morceau de viande prélevé a été introduit dans un sachet alimentaire en plastique étiqueté et placé dans une glacière contenant des packs réfrigérants.

\section{Aliments distribués}

Les aliments distribués aux animaux sont de deux types; l'aliment «granulé » et l'aliment « classique ». L'aliment granulé a été constitué à partir de fourrage $(70 \%)$ séché et broyé puis mélangé à d'autres produits alimentaires. Le mélange a été ensuite passé sous une presse à granulé. L'aliment classique est l'aliment mise au point par Mensah et Ekue (2003) lors de la vulgarisation de l'aulacodiculture. Il est constitué à $70 \%$ de fourrage vert et d'un complément aliment (30\%) dont la composition chimique est indiquée dans le Tableau 1.

\section{Etude des qualités sensorielles et organoleptiques de la viande d'aulacode \\ Pour la détermination de la qualité organoleptique, une présélection au hasard de}


18 panélistes parmi les volontaires ayant déjà consommé ou non la viande d'aulacode a été effectuée. Ces panélistes ont, par la suite, été soumis à des séances d'entraînement qui ont été conduites sur deux jours. Après ces deux jours, 12 panélistes définitifs ont été retenus pour le test à trois répétitions. L'appréciation des caractéristiques physiques de la viande s'est faite par des tests de fraîcheur (couleur et texture) et par des tests de cuisson (la flaveur, la jutosité et la tendreté). La couleur et la texture ont été déterminées sur la viande fraîche. Ensuite, six morceaux de viande de 30 g environ ont été prélevés sur chaque membre postérieur. Afin de conserver leur intégrité, chaque morceau de viande a été introduit dans un double sachet alimentaire en plastique étiqueté. Ensuite, les sachets alimentaires contenant les morceaux de viande ont été mis dans des casseroles en aluminium neuves à moitié remplie d'eau pour les cuire. La cuisson a été faite au même moment sur deux plaques chauffantes de marque SAYONA et de puissance $1500 \mathrm{~W}$, réglées à la même température $\left(100{ }^{\circ} \mathrm{C}\right)$ pendant $42 \mathrm{~min}$. Aucun assaisonnement n'a été effectué. A la fin de la cuisson, les sachets ont été pesés, puis vidés de leur exsudat, ils ont été à nouveau pesés. Ceci a donc permis d'évaluer la jutosité en poche de cuisson. Ensuite, la flaveur, la jutosité et la tendreté ont été évaluées selon Cartier et Moëvi (2007) et Coibion (2008) sur une échelle de 1 à 4 (Tableau 2). L'étude s'est déroulée entre $9 \mathrm{~h}$ et $12 \mathrm{~h}$ dans une salle parfaitement close, climatisée et éclairée avec des ampoules Néon. Les panélistes ont été disposés de sorte à éviter toute forme de communication. Aucun morceau de viande n'a été dégluti après mastication et le passage d'une étape à une autre a nécessité le rinçage de la bouche avec de l'eau minérale.

\section{Analyse biochimique de la viande}

Les constituants biochimiques tels que le taux d'humidité ( $\mathrm{H} \%)$, le taux de matière sèche (MS\%), le taux de protéines brutes, le taux de matière grasse et le taux de cendre brute ont été tous déterminés selon les méthodes de BIPEA (1976) et de AOAC (1995).

\section{Analyse statistique}

L'analyse statistique a été réalisée avec le logiciel Statistica version 7.1. Ce logiciel a permis de réaliser les calculs des moyennes et des écart-types. Le test de Kruskal-Wallis a été utilisé pour traiter les données relatives aux caractéristiques chimiques des aliments.

\section{RESULTATS}

\section{Caractéristiques organoleptiques de la viande d'aulacode}

Les résultats concernant les caractéristiques organoleptiques sont consignés dans le Tableau 3 . Le choix des panélistes a permis d'affirmer que la viande des aulacodes nourris à l'aliment classique a une coloration moins foncée que celle de ceux nourris à l'aliment granulé. Pour ces viandes, la coloration est respectivement « rosé-claire » et «rosé-rouge». Selon les panélistes, la viande d'aulacode est juteuse, tendre avec une texture «grossière » et une flaveur prononcée quel que soit le type d'aliment distribué. Les résultats de la jutosité en poche de cuisson (environ 25\%) confirme le choix des panélistes qui ont montré que la viande d'aulacode est juteuse (Tableau 4).

L'analyse des résultats relatif à la préférence des panelistes indique que la viande des aulacodes nourris au granulé présente les pourcentages les plus élevés sauf pour la tendreté où les valeurs sont équivalentes (Tableau 5).

La Figure 1 illustre la représentation du profil sensoriel de la viande d'aulacode. La couleur des viandes, leurs textures et leurs jutosités ont connu des variations en fonction de l'aliment. Cependant, la flaveur et la tendreté ne semblent pas affectées par le régime alimentaire des aulacodes.

\section{Caractéristiques biochimiques}

Les caractéristiques biochimiques de la viande d'aulacode sont présentées dans le Tableau 4. L'analyse des valeurs moyennes de matière sèche, de protéines brutes, de matière grasse et de cendres brutes ne montrent aucune différence significative $(\mathrm{P} \geq 0,05)$ selon les deux types d'aliments. 
Tableau 1 : Composition des différents types d'aliments testés chez les aulacodes.

\begin{tabular}{lcc}
\hline & Aliment Granulé & Aliment Classique \\
\hline Matière sèche (\%) & 92,05 & 86,68 \\
Protéines brutes (\% MS) & 18,8 & 11,52 \\
Matière grasse (\% MS) & 2,46 & 2,19 \\
Fibres brutes (\% MS) & 30,12 & 31,9 \\
Cendres brutes (\% MS) & 7,91 & 10,43 \\
Calcium (g/kg MS) & 5,23 & 3,2 \\
Phosphore (g/kg MS) & 4,21 & 2,6 \\
Energie brute (kcal/kg MS) & 4218,32 & 4152,9 \\
\hline
\end{tabular}

Tableau 2 : Grille d'évaluation sensorielle de la viande.

\begin{tabular}{llllll}
\hline \multicolumn{5}{c}{ Paramètres sensoriels } \\
\hline Grille & Couleur & Texture & Justosité & Tendreté & Flaveur \\
\hline 1 & Blanc & Très fine & Très sec & Très dure & Très faible \\
2 & Rosé-clair & Fine & Sec & Dure & Faible \\
3 & Rosé-rouge & Grossière & Juteux & Tendre & Prononcée \\
4 & Rouge & Très grossière & Très juteux & Très tendre & Très prononcée \\
\hline
\end{tabular}

Tableau 3: Caractéristiques organoleptiques de la viande d'aulacode en fonction du régime alimentaire.

\begin{tabular}{llcc}
\hline Paramètres & & Aliment Granulé (\%) & Aliment classique (\%) \\
\hline \multirow{3}{*}{ Couleur } & Blanc & 2,78 & 5,56 \\
& Rosé-clair & 13,89 & 50,00 \\
& Rosé-rouge & 50,00 & 36,11 \\
& Rouge & 33,33 & 8,33 \\
\hline \multirow{3}{*}{ Texture } & Très fine & 0 & 13,89 \\
& Fine & 36,11 & 36,11 \\
& Grossière & 55,56 & 44,44 \\
& Très grossière & 11,11 & 5,56 \\
\hline \multirow{3}{*}{ Jutosité buccale } & Sec & 2,78 & 2,78 \\
& Juteux & 19,44 & 33,33 \\
& Très juteux & 44,44 & 44,44 \\
& Très dure & 27,78 & 19,44 \\
\hline \multirow{3}{*}{ Tendreté } & Dure & 8,33 & 5,56 \\
& Tendre & 16,67 & 19,44 \\
& & 47,22 & 52,78
\end{tabular}




\begin{tabular}{llcc} 
& Très tendre & 27,78 & 22,22 \\
\hline \multirow{3}{*}{ Flaveur } & Très faible & 2,78 & 0 \\
& Faible & 19,44 & 33,33 \\
& Prononcée & 69,44 & 52,78 \\
& Très prononcée & 5,56 & 13,89 \\
\hline
\end{tabular}

Tableau 4 : Jutosité en poche de cuisson de la viande d'aulacode en fonction du régime alimentaire.

\begin{tabular}{ccc}
\hline & Viande des animaux du Granulé & Viande des animaux de l'Aliment classique \\
\hline $\begin{array}{c}\text { Jutosité moy } \\
(\%)\end{array}$ & $24,72 \pm 13,71 \mathbf{a}$ & $25,34 \pm 10,95 \mathbf{a}$ \\
\hline
\end{tabular}

Les valeurs moyennes sur une même ligne portant des lettres différentes sont significativement différentes $(\mathrm{p} \geq 0,05)$.

Tableau 5 : Préférence des panélistes en fonction du régime alimentaire.

\begin{tabular}{llccccc}
\hline & \multicolumn{2}{c}{ Granulé } & \multicolumn{2}{c}{ Aliment Classique } & \multicolumn{2}{c}{ Abstention } \\
\cline { 2 - 7 } Paramètres & Effectifs & Pourcentage (\%) & Effectifs & Pourcentage (\%) & Effectifs & Pourcentage (\%) \\
& & & & & & \\
\\
\hline Couleur & 16 & 44,44 & 12 & 33,33 & 8 & 22,22 \\
Texture & 15 & 41,67 & 13 & 36,11 & 8 & 22,22 \\
Jutosité & 17 & 47,22 & 16 & 44,44 & 3 & 8,33 \\
Tendreté & 16 & 44,44 & 16 & 44,44 & 4 & 11,11 \\
Flaveur & 19 & 52,78 & 15 & 41,67 & 2 & 5,56 \\
\hline
\end{tabular}

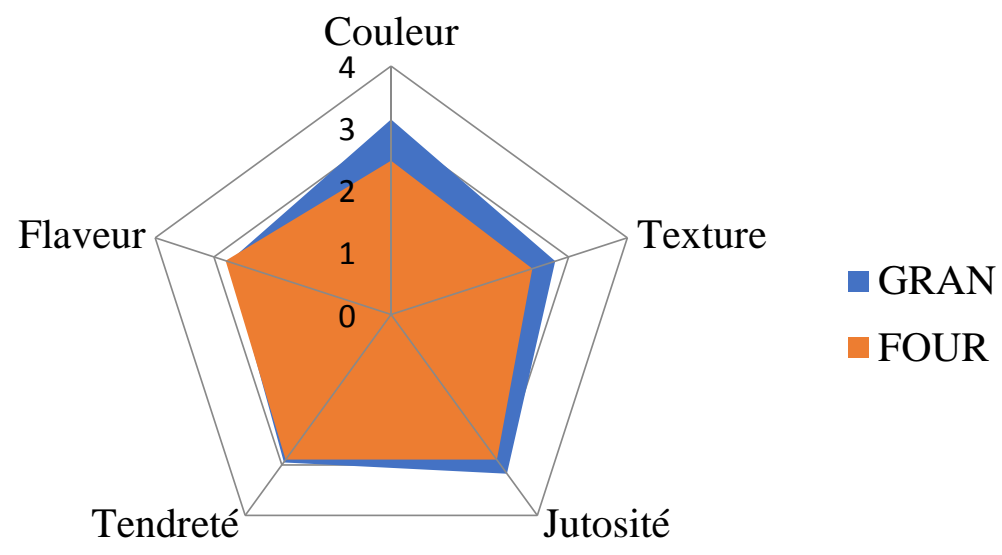

GRAN = viande d'aulacode nourris avec l'aliment granulé et FOUR = Viande d'aulacode nourris avec l'aliment classique)

Figure 1 : Profil sensoriel de la viande d'aulacode selon le type d'aliment. 


\section{DISCUSSION}

Les paramètres organoleptiques retenus dans le cadre de ce travail sont identiques à ceux utilisés par Coibion (2008). Aucune différence significative n'a été enregistré pour la couleur, la texture et la jutosité au cours de nos travaux. Eu égard aux choix des panélistes, il peut être admis que la viande des aulacodes d'élevage nourris au granulé présente une coloration plus foncée (Rosé-Rouge) que celle des animaux nourris à l'aliment classique (Rosée-clair). Cette différence de coloration pourrait s'expliquer par la composition des types d'aliments. En effet, les rations alimentaires utilisées (aliment granulé et aliment classique) sont différentes du fait des ingrédients qui les composent. Les travaux conduit par Mohamed et al. (2008) ont montré que la nature de l'aliment distribué influençait également la coloration de la viande. En effet, ils ont déterminé que les animaux engraissés au fourrage donnaient des viandes plus sombres que ceux dont la finition était réalisée avec des aliments concentrés chez les ruminants. Les résultats obtenus dans le cadre de cette étude sont contraires à leur résultat car c'est la viande des animaux nourris au granulé (aliment concentré) qui était plus sombre. La viande de ce rongeur a été reconnue juteuse par $44,44 \%$ des panélistes. Selon Micol et al., (2010), il existe une jutosité initiale perçue au premier coup de dent essentiellement liée à la quantité d'eau présente dans la viande et une secondaire jutosité, en relation avec la teneur en lipides de celle-ci. Renand et al. (2008) affirment qu'une faible teneur de gras intramusculaire limiterait la jutosité. La première serait donc à l'origine de la jutosité perçue dans la viande d'aulacode. Bien que la préférence de tendreté des viandes des deux types de rations soit identique en pourcentage $(44,44 \%)$, cependant, une différence est constatée pour la tendreté de la viande issue des aulacodes nourris à l'aliment classique $(52,78 \%)$ contre celle pour les animaux nourris au granulé $(47,22 \%)$. Cette différence de tendreté enregistrée pourrait être liée à la composition de la viande tant en fibres et en tissu conjonctif. D'après Ouali et al. (2004), l'augmentation de la tendreté de la viande est liée à un affaiblissement de la structure myofibrillaire grâce à une dégradation des protéines de structure et des liaisons intermoléculaires sous l'action d'enzymes endogènes. Certains auteurs tel que Lebret (2008) ont montré que l'alimentation influence la tendreté de la viande chez le porc. Plus de la moitié des panélistes ont affirmé que la viande d'aulacode, quel que soit le régime, a une flaveur prononcée. La préférence de leur viande par les consommateurs est due sans doute à la flaveur qui procure un arôme particulier. Aussi, environ 52,78\% préfèrent la viande des animaux nourris au granulé. Selon Cartier et Moëvi (2007) la conduite alimentaire des animaux influencerait la flaveur des viandes. Certains affirment que la flaveur dépend essentiellement de la teneur en lipides intramusculaires (Lebret et al., 2015) or la teneur en matière grasse de la viande obtenue dans cette étude est faible et statistiquement identique.

L'analyse de nos résultats sur les paramètres chimiques n'a révélée aucune différence significative par rapport aux deux rations. La viande d'aulacodes nourris au granulé a un taux d'humidité de $73,62 \%$ et celui de ceux nourris à l'aliment classique a été de 75,64\%. Ces résultats sont conformes à ceux de Penda (2009). En effet cet auteur a enregistré un taux d'humidité de $77 \%$ avec des aulacodines et un taux de $75 \%$ avec des aulacodins. Cependant de faibles taux d'humidité, compris entre 48 et $49 \%$, ont été obtenus par Wogar et al. (2013) lors de son étude sur les aulacodes. Chez le lapin, un faible taux d'humidité $(67,46 \%)$ a été rapporté par Gigaud et al. (2007). Ces observations révèlent que la viande d'aulacode est plus riche en eau que celle du lapin. Les taux protéiques obtenus lors de nos travaux $(22,34 \%$ avec l'aliment granulé et $21,14 \%$ avec l'aliment classique) sont proches de ceux obtenus par Wogar et al. 
(2013). En fonction du niveau énergétique de la ration, cet auteur a enregistré des taux protéiques variant entre 18,78 et $21,12 \%$. Selon ses travaux, le niveau protéinique de la viande serait fonction de la valeur énergétique de la ration. La variation de la teneur en protéine de la viande est fonction du type de muscle, de la race et du sexe de l'animal (Penda, 2009; Salifou et al., 2013). En ce qui concerne la matière grasse, des proportions de 1,4 et 1,13\% ont été obtenues respectivement avec le granulé et l'aliment classique. Nteme Ella et al. (2014) ont enregistré des taux similaires aux valeurs obtenues au cours de cette étude. Selon Penda (2009), la viande d'aulacode est une viande pauvre en matières grasses. Cependant, les travaux de Wogar et al. (2013) enregistrent des valeurs plus élevées qui varient entre 11,92 et $12,64 \%$ dans la viande d'aulacodes adultes. La graisse contenue dans la viande rouge varie généralement selon l'espèce, le format, l'âge, le régime alimentaire et le muscle (Bauchart et al., 2008 ; Nteme-Ella et al., 2009). Les taux de cendres brutes enregistrés au cours de nos travaux sont supérieurs à ceux obtenus par Nteme Ella et al. (2014) qui ont obtenu un taux moyen de $1 \%$. Ils sont également supérieurs à ceux de Penda (2009) qui a enregistré des taux moyens compris entre 0,84 et $1,36 \%$. Ces valeurs élevées de taux de cendre observées au cours de nos travaux, pourraient s'expliquer par la complémentation minérale dont bénéficiaient nos aulacodes.

\section{Conclusion}

Cette étude a permis de montrer que la viande d'aulacode est une viande à coloration rosé-rouge (rouge) et à texture grossière. Elle est tendre et présente une flaveur prononcée. Quant à l'analyse biochimique de la viande, il est à noter que la composition chimique de la viande d'aulacode ne varie pas de manière significative, selon le régime. Pour la majorité des paramètres organoleptiques, la viande des aulacodes nourris au granulé est préférée à celle de ceux nourris à l'aliment classique. Il serait donc impérieux d'envisager une sélection sur la base des paramètres zootechniques et génétique mais aussi en tenant compte de l'alimentation pour satisfaire les consommateurs.

\section{CONFLIT D'INTERETS}

Les auteurs déclarent ne pas avoir de conflit d'intérêts.

\section{CONTRIBUTIONS DES AUTEURS}

SS a conçu l'étude, a participé à la collecte des données et à la rédaction du manuscrit. AAY a participé à la rédaction du manuscrit, acquisition de données (travaux de laboratoire) et leur interprétation. BT a participé à la rédaction du manuscrit, à l'analyse statistique ainsi qu'à l'interprétation des résultats. AF a supervisé les travaux et participé à la rédaction du manuscrit. Tous les auteurs ont lu et approuvé le manuscrit final.

\section{REMERCIEMENTS}

Cette étude a été réalisée dans le cadre du consortium Afrique One « Ecosystem and Population Health: Expanding Frontiers in Health » et financé par le Wellcome Trust (WT087535MA) d'une part et par l'UEMOA d'autre part.

\section{RÉFÉRENCES}

AOAC. 1995. The Association of Official Analytical Chemists. Official Methods of Analyses (16th edn. 5th revision). VA. AOAC International. Arlington (USA).

Bauchart D, Chantelot F, Gandemer G. 2008. Qualités nutritionnelles de la viande et des abats chez le bovin : données récentes sur les principaux constituants d'intérêt nutritionnel. Cah. Nutr. Diététique, 43 : 29-39. DOI : CND-05-2008-43-HS10007-9960-101019-200805850

BIPEA, 1976, Bureau Interprofessionnel d'Etudes analytiques. Recueil des méthodes d'analyses des communautés Européennes. BIPEA : 2 route du port Charbonnier, 92230 Gennevilliers. 
Cartier P, Moëvi I. 2007. Le Point sur la Qualité des Carcasses et des Viandes de Gros Bovins. Institut de l'Élevage : Paris.

Cassignol V. 2018. Facteurs déterminant la qualité sensorielle de la viande bovine : Quelle importance de la race ? Viandes et Produits Carnés, 1(5): 1-10. DOI : http://viandesetproduitscarnes.com/phoc adownload/vpc_vol_34/3415_cassignol_ race_et_qualite_sensorielle_viande_bovi ne.pdf

Coibion L. 2008. Acquisition des qualités organoleptiques de la viande bovine : adaptation à la demande du consommateur. Mémoire pour l'obtention du grade de Docteur vétérinaire, Ecole nationale vétérinaire de Toulouse ; p. 97.

Gigaud V, Combes S. 2007. Les atouts nutritionnels de la viande de lapin : comparaison avec les autres produits carnés. $12^{\text {ème }}$ Journée de la recherche cunicole, Le Mans, France; 187-190. DOI :

https://www.cuniculture.info/Docs/Maga zine/Magazine2007/Fichiers.pdf/JRC2007/7-Qualite/2q-gigaud.pdf

Lebret B, Prache S, Berri C, Lefèvre F, Bauchart D, Picard B, Corraze G, Médale F, Faure J, Alami-Durante H. 2015. Qualités des viandes : influences des caractéristiques des animaux et de leurs conditions d'élevage. INRA Prod. Anim., 28(2). 151-168. DOI : https://doi.org/10.20870/productionsanimales.2015.28.2.3022

Lebret B. 2008. Effects of feeding and rearing systems on growth, carcass composition and meat quality in pigs. Animal., 2 (10). 1548-1558.

DOI: https://doi.org/10.1017/S17517311 08002796

Mensah GA, Ékué MRM. 2003. L'essentiel en aulacodiculture. C.B.D.D./NCIUCN/ KIT, énin/Royaume des Pays-Bas.
Micol D, Jurie C, Hocquette JF. 2010. Qualités sensorielles de la viande bovine. Impacts des facteurs d'élevage ? In : Bauchard D., Picard B., Muscle et viande de ruminant. QUAE (éditions). Paris; 163-169.

Mohamed A, Jamilah B, Abbas KA., Abdul Rahman R. 2008. A review on some factors affecting colour of fresh beef cuts. J. Food Agric. Env., 6 :181-186. DOI : https://agris.fao.org/agrissearch/search.do?recordID=US20130159 1891

Niaga M. 2002. L'aulacode au Sénégal : Etat des connaissances auprès des ruraux et des consommateurs ; Résultats d'un élevage expérimental à Mako, région de Tambacouda. Thèse de Médecine Vétérinaire, Dakar, p. 135.

Nteme Ella GS, Aoussi A, Penda R, Fantodji A, Missohou A, Bakou S. 2009. Composition chimique des muscles du membre pelvien du grand aulacode (Thryonomys swinderianus, Temminck 1827). Revue Africaine de Santé et de Productions Animales, 7 (1) : 29-32. DOI : https://www.researchgate.net/publication 1288184564

Nteme Ella GS, Aoussi S, Kouame P, Fantodji A, Bakou S. 2014. Détermination de la couleur et de la valeur nutritionnelle de la viande d'aulacodes sauvages (Thryonomys swinderianus, Temminck, 1827). Int. J. Biol. Chem. Sci., 8 (2) : 610620. DOI: http://ajol.info/index.php/ijbcs Ouali A., Herrera-Mendez CH, Coulis G, Becila S, Boudjellal A, Aubry L, Sentandreu MA. 2006. Revisiting the conversion of muscle into meat and the underlying mechanisms. Meat Sci, 74 : 44-58.

DOI : https://doi.org/10.1016/j.meatsci.2006.05 .010

Penda RE. 2009. Contribution à l'étude de la qualité de la viande d'aulacode (Thryonomys swinderianus, Temminck, 
1827) : caractérisation de la composition chimique et de la couleur des muscles du membre pelvien. Mémoire de diplôme de master II en Qualité des Aliments de l'Homme, Ecole Inter - Etats des Sciences et Médecine Vétérinaires, Dakar, p. 40.

Renand G, Havy A, Turin F. 2002. Caractérisation des aptitudes bouchères et qualités de la viande de trois systèmes de production de viande bovine à partir des races rustiques françaises Salers, Aubrac et Gasconne. INRA Prod. Anim., 15 (3) : 171-183.

DOI :

https://www6.inrae.fr/productionsanimales/content/download/3885/40098/ version/1/file/Prod_Anim_2002_15_3_0 3.pdf

Salifou CFA, Youssao AKI, Ahounou GS, Tougan PU, Farougou S, Mensah GA, Clinquart A. 2013. Critères d'appréciation et facteurs de variation des caractéristiques de la carcasse et de qualité de la viande bovine. Ann. Méd. Vét., 157: 27-42. DOI: http://www.facmv.ulg.ac.be/amv/articles /2013_157_1_03.pdf
Soro S, Karamoko Y, Soro D, Gonnety TJ; Fantodji A. 2014. Contribution of young grass-cutter (Thryonomys swinderianus) feeding after weaning. Les Technologies de Laboratoire, 8 (34) : 145-152. DOI : https://www.academia.edu/20140356/Co ntribution_of_young_grass-

cutter_Thryonomys_swinderianus_feedi ng_after_weaning

Traoré B, Mensah GA, Fantodji A. 2009. Influence de la forme physique des aliments sur la croissance et le rendement en carcasse de Thryonomys swinderianus à trois stades physiologiques. Bul Rec Agr Bénin, 65: 1-31. DOI : https://www.researchgate.net/publication /261704668

Wogar GSI, Ufot ML, Henry AJ, Inyang IE, Efe EE. 2013. Composition and Emulsifying Characteristics of Grasscutter Meat from Varying Dietary Energy Levels. Journal of Agricultural Science; 5(1): 314-318. DOI: 10.5539/jas.v5n1p314. 